the remaining organic compounds which form the second, "biological" peak.

With a sterile sample a peak ratio of about 500 to 1 would be expected. The result from the Martian soil was about 75 to 1 due to the high "biological" peak. This is several times more active than non-sterilised soils from the dry valleys of Antarctica. The result from the control experiment with heat-sterilised soil is crucial in this instance, a high peak ratio indicating the possibility that biological rather than chemical processes capable of fixing atmospheric carbon monoxide or dioxide in the presence of light are taking place in the soil. If life exists on Mars, this is what might be expected in the soil surface which is strongly illuminated and bathed in an atmosphere of about $95 \%$ carbon dioxide. The biologists would be even more encouraged if organic compounds were detected in the Martian soil.

Unfortunately, the initial run of the organic chemistry analysis was less than satisfactory due to the temperamental soil sampling boom which apparently failed to deliver a full load of soil to the gas chromatograph-mass spectrometer. No organic compounds were detected. A full soil load has now been obtained and a further analysis is being carried out. If organic compounds are not detected this would be a blow, though not a fatal one, to the concept of life on Mars, and would render interpretation of the three biology experiments more difficult.

\title{
USSR SPACE
}

\section{Forward with the programme}

Vera Rich reports on the latest Soviet space missions

"Soviet science considers the creation of orbital space stations with interchangeable crews as the main highway of mankind into space. They can form "cosmodromes in space', launch pads for flights to other planets. Large-scale scientific laboratories will arise for the investigation of space technology and biology, medicine and geophysics, astronomy and astrophysics."

So said Mr Brezhnev in a recent statement. The idea was by no means new; it is a fundamental tenet of all Tsiolkovskii's work that such a station would and must precede manned exploration of the moon and planets. There are, indeed, some indications that Soviet space planners have at times been prepared to consider the alternative of manned exploration direct from earth-the argument that a lunokhod-carrying automatic spacecraft could carry out its survey work at "half the cost" of a manned mission, for example, suggests that the latter possibility had at least reached the costing stage. Now, the flight of the unmanned Luna 24 has repeated the task of Luna 16 by obtaining and returning with a sample of moon-rock, though from a depth of two metres and from Mare crisium, from which no samples had yet been obtained. Presumably present space plans still do not envisage cosmonauts venturing beyond earth orbit.

Whatever the overall timetable of the Soviet Union's space plan, though, many of the experiments now being conducted by the 7-week-old Salyut 5 mission suggest that the concept of a permanently manned space station is still well to the fore. These latter experiments fall into three main groups: geophysical and astrophysical observations, technological experiments and biological experiments. Of the technological experiments, some are directly concerned with processes and techni- ques which would be required in the construction of a space station-notably the study of the behaviour of gases in liquids in space and, of some importance, a soldering experiment. Unlike the welding experiments of an earlier mission, the soldening, which uses a highly exothermal chemical reaction to produce the necessary heat, is aimed 2 less at itesting the cosmonaut's dexterity than at investigating the process itself. The welded metal specimens are to be returned to earth for further investigation of their structure.

The emphasis has clearly shifted to the properties of materials treated under conditions of weightlessness. Other experiments include the growing of crystals (also to be returned to earth), and the cooling of molten metals - bismuth, lead, tin and cadmium-to determine surface tension. These experiments will, of course, provide data of considerable theoretical significance: crystals grown under weightlessness, for example, should be free of convection-induced defects, and should, it is hoped, provide valuable comparative data for crystallographers and geologists. It is significant, however, that all these experiments are described in the official announcements as "technological", suggesting that their practical importance should not be minimised.

The medical problems of prolonged spaceflight are also receiving considerable attention. Apart from such obvious parameters as muscle tone, cardiovascular and respiratory performance, and loss of body weight-measured on the special "massometer"--the medical programme includes special monitoring of the cosmonauts in a "vertical" position (however that is to be interpreted), tests on reaction time in performing complex operations and manual control (real or simulated) of the spacecraft (the simulated control presumably relates to possible emergencies), and even possible variations in taste.

\section{Sorry, for copyright \\ reasons some images on this page may not be available online}

\section{Before blast-off for Salyut 5}

Press comment on the medical programme suggests a growing concern over the psychological effects of prolonged spaceflight. These would arise from a lack of external stimuli, the restriction of living space and general monotony-an aspect of spaceflight which will assume ever greater importance in future missions when the length of flight and the crew size increases. Aboard Salyut 5 are such simple prophylactics as recorded music (from folk to classical) and a varying make-up of the ration-packs.

Commenting on the radiation safety system used aboard the Salyut craft, Ludvig Palmbakh of the USSR Institute of General Genetics stated that the protective measures used are "well adapted" for work near Earth, since, if solar radiation rises to danger level, the crew can be quickly evacuated and brought back. For deepspace missions, however, "more active" means of protection would probably be required, possibly involving the deflection of high-enengy particles by electromagnetic fields. The design of such systems entails considerable technical difficulty, so the fullest possible information is required both on the nature of the radiation and the actual hazard involved. The cytological and genetic experiments, using drosophila and hawksbeard, carried by Salyut 5 , should provide further data on these hazards. 